\title{
Prediction of response to preoperative chemoradiotherapy and establishment of individualized therapy in advanced rectal cancer
}

\author{
TOSHIHIRO NAKAO, TAKASHI IWATA, MASANORI HOTCHI, KOZO YOSHIKAWA, JUN HIGASHIJIMA, \\ MASAAKI NISHI, CHIE TAKASU, SHOHEI ETO, HIROKI TERAOKU and MITSUO SHIMADA \\ Department of Surgery, Tokushima University, Tokushima 770-8503, Japan
}

Received January 13, 2015; Accepted March 9, 2015

DOI: $10.3892 /$ or.2015.4196

\begin{abstract}
Preoperative chemoradiotherapy (CRT) has become the standard treatment for patients with locally advanced rectal cancer. However, no specific biomarker has been identified to predict a response to preoperative CRT. The aim of the present study was to assess the gene expression patterns of patients with advanced rectal cancer to predict their responses to preoperative CRT. Fifty-nine rectal cancer patients were subjected to preoperative CRT. Patients were randomly assigned to receive CRT with tegafur/gimeracil/oteracil (S-1 group, $\mathrm{n}=30$ ) or tegafur-uracil (UFT group, $n=29$ ). Gene expression changes were studied with cDNA and miRNA microarray. The association between gene expression and response to CRT was evaluated. cDNA microarray showed that 184 genes were significantly differentially expressed between the responders and the non-responders in the S-1 group. Comparatively, 193 genes were significantly differentially expressed in the responders in the UFT group. TBX18 upregulation was common to both groups whereas BTNL8, LOC375010, ADH1B, HRASLS2, LOC284232, GCNT3 and ALDH1A2 were significantly differentially lower in both groups when compared with the non-responders. Using miRNA microarray, we found that 7 and 16 genes were significantly differentially expressed between the responders and non-responders in the S-1 and UFT groups, respectively. miR-223 was significantly higher in the responders in the S-1 group and tended to be higher in the responders in the UFT group. The present study identified several genes likely to be useful for establishing individualized therapies for patients with rectal cancer.
\end{abstract}

\section{Introduction}

Patients with locally advanced rectal cancer are routinely treated with preoperative chemoradiotherapy (CRT). Randomized

Correspondence to: Dr Toshihiro Nakao, Department of Surgery, Tokushima University, 3-18-15 Kuramoto-cho, Tokushima 770-8503, Japan

E-mail: nakao.toshihiro@tokushima-u.ac.jp

Key words: chemoradiotherapy, rectal cancer, microarray, miRNA, miR-223 phase III trials have previously shown that preoperative CRT significantly improves the local control of cancer as compared with preoperative radiation alone or postoperative CRT (1-3). We previously performed a phase I clinical study and determined the recommended dose $\left(80 \mathrm{mg} / \mathrm{m}^{2}\right)$ of tegafur/gimeracil/ oteracil (S-1) for preoperative CRT (4). We then undertook a phase II clinical study to evaluate the efficacy and toxicity of preoperative CRT using tegafur-uracil (UFT; $300 \mathrm{mg} / \mathrm{m}^{2} /$ day) vs. S-1 $\left(80 \mathrm{mg} / \mathrm{m}^{2} /\right.$ day) in 59 patients with locally advanced rectal cancer (5). This is the first study to use $\mathrm{S}-1$ as a single agent for CRT of locally advanced rectal cancer. Using the Response Evaluation Criteria in Solid Tumors (RECIST) guidelines, we found no statistically significant difference in terms of the patient response rate $(p=0.52)$ between the $S-1$ and UFT groups, with no difference in the downstaging rate, resection of tumor, sphincter preservation and marginal invasion. The only significant finding was that the incidence of grade 3 diarrhea was significantly more frequent in the S-1 group $(7 \%)$ as compared with the UFT group $(0 \%)(\mathrm{p}=0.02)$.

Although this is an overall positive result, patient responses to preoperative CRT differ between individuals. Some studies have therefore sought to investigate the gene expression profiles in patients with rectal cancer to predict their response to preoperative CRT (6-9). However, as yet, there are no specific biomarkers with which to predict these responses. Therefore, it is becoming increasingly important to identify predictive biomarkers of preoperative CRT.

Fluorouracil-based regimens have been generally used for preoperative CRT in patients with rectal cancer and some studies have reported the efficacy of oral fluorouracil prodrugs, such as UFT, S-1 and capecitabine (10-13). However, the best agent for preoperative CRT in patients with rectal cancer has not yet been determined.

Here, we employed cDNA and miRNA microarray analyses to screen for biomarkers that could be useful in predicting the responses of patients with rectal cancer to preoperative CRT following treatment with S-1 or UFT, with the later hope that these biomarkers can be used to tailor therapies for patients with rectal cancer.

\section{Materials and methods}

Patients. From April 2008 to October 2010, 62 patients with locally advanced rectal cancer within $10 \mathrm{~cm}$ from the anal 


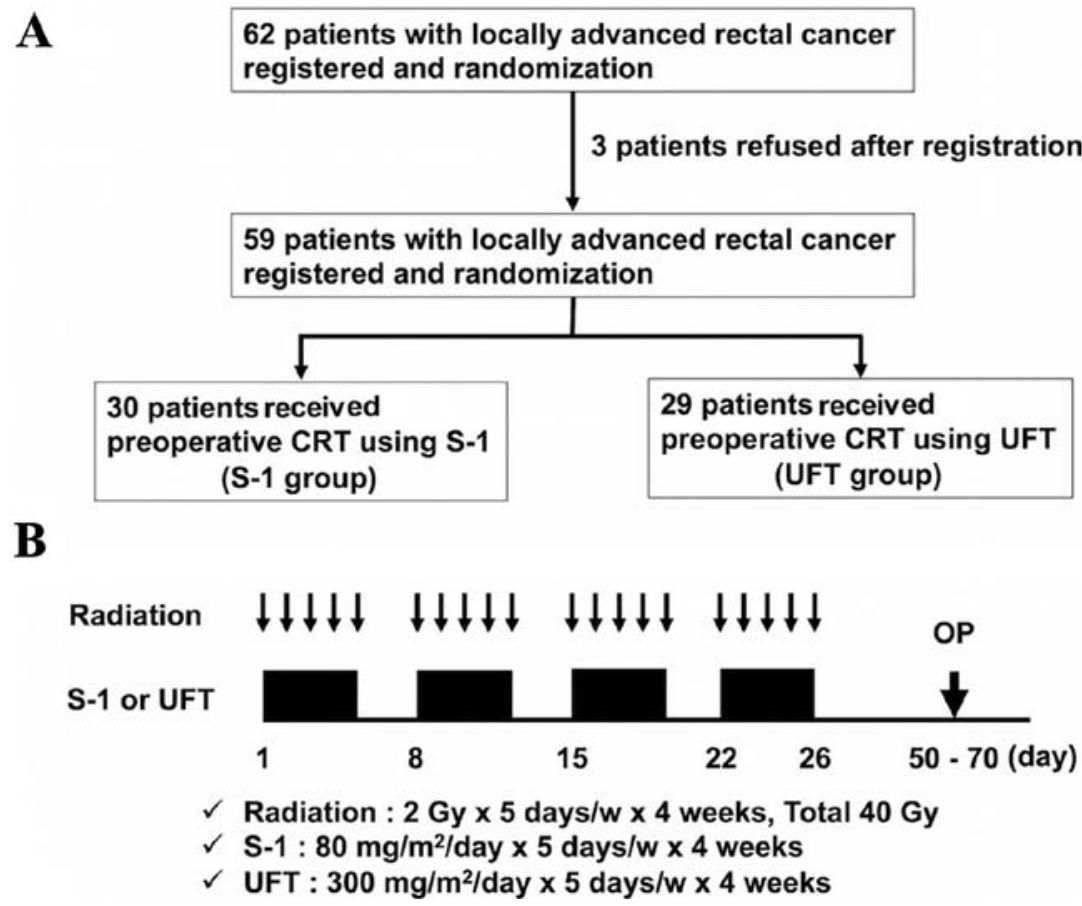

Figure 1. (A) Diagram illustrating patient enrollment, randomization and treatment schedule. Sixty-two patients with locally advanced rectal cancer registered for the present study. Three patients refused to participate, thus 59 patients were randomly assigned to the $\mathrm{S}-1$ group (tegafur/gimeracil/oteracil; $\mathrm{n}=30$ ) or the UFT group (tegafur-uracil; n=29). (B) Design of preoperative chemoradiotherapy (CRT). Strong radiation at 2 Gy was performed for 5 days/week for 4 weeks, with S-1 $\left(80 \mathrm{mg} / \mathrm{m}^{2}\right)$ or UFT $\left(300 \mathrm{mg} / \mathrm{m}^{2}\right)$ administered orally on the same day. The conventional four-field box radiotherapy technique was used.

verge were enrolled in the present study across multiple institutions. Patients were randomly assigned to receive preoperative CRT with S-1 (S-1 group) or preoperative CRT with UFT (UFT group). Despite signing the consent form, three patients chose to leave the study before treatment, and they were treated surgically without CRT (Fig. 1). Finally, 59 patients (S-1 group, $n=30$; UFT group, $n=29$ ) were confirmed, each fulfilling the following criteria: i) Eastern Cooperative Oncology group (ECOG) performance status of $0-2$; ii) white blood count $\geq 4,000 / \mathrm{mm}^{3}$; iii) platelet count $\geq 100,000 / \mathrm{mm}^{3}$; iv) serum total bilirubin $<1.5 \mathrm{mg} / \mathrm{dl}$; v) creatinine $<1.5 \mathrm{mg} / \mathrm{dl}$; and vi) heart function (stable cardiac rhythm, no active angina, no clinical evidence of congestive heart failure). Informed consent was obtained from all patients included in this study, and the present study protocol was approved by our local ethics committees. There was no statistically significant difference in clinicopathological features between the S-1 and UFT group except for gender (data not shown).

For gene expression profiling, biopsy specimens were prospectively collected during colonoscopic examination before commencing preoperative CRT. These samples were used for RNA extraction when parallel specimens contained at least $70 \%$ tumor cells. Samples were snap frozen immediately in liquid nitrogen and stored at $-80^{\circ} \mathrm{C}$ until RNA extraction.

Preoperative CRT. Preoperative CRT is routinely prescribed for patients with locally advanced cancers ( $\geq \mathrm{T} 3$ and/or $\geq N 1$ ) or for those who have very distal T2N0 cancers close to or involving the sphincter. Patients received CRT with a total dose of 40 Gy of pelvic irradiation, which was administered five times per week, with a daily fraction of $2 \mathrm{~Gy}$ using a four-field technique. The superior margin of the radiation field was the bifurcation point of the aorta, whereas the inferior margin was marked at least $4 \mathrm{~cm}$ below the tumor. Radiation was delivered concomitantly with $\mathrm{S}-1\left(80 \mathrm{mg} / \mathrm{m}^{2}\right)$ or UFT $\left(300 \mathrm{mg} / \mathrm{m}^{2}\right)$. If grade 3 toxicity occurred, the dose of S-1 or UFT was reduced to $75 \%$ of the previous dose. If the neutrophil count decreased to $<1,500 / 1$ or the platelet count decreased to $<100,000 / 1$, chemotherapy was stopped until recovery. Surgery was performed 6-8 weeks after completion of preoperative CRT. The design and timeline of preoperative CRT administration is shown in Fig. 1.

cDNA microarray. cDNA and miRNA microarray (described below) were performed at Hokkaido System Science Co., Ltd. (Hokkaido, Japan). High-quality RNA (50-200 ng) was amplified and cyanine 3-CTP labeled with the One Color Low Input Quick Amp Labeling kit (version 6.5; Agilent Technologies, Santa Clara, CA, USA) according to the manufacturer's instructions. Labeled and amplified RNA was purified with Qiagen RNeasy Mini spin columns (Qiagen, Valencia, CA, USA). RNA labeling efficiency was controlled using the NanoDrop spectrophotometer (Thermo Scientific, Wilmington, DE, USA). Labeled cRNA (600 ng) was fragmented and hybridized to the Whole Human Genome Expression Array G4851A (8x60K; Agilent) according to the manufacturer's instructions. In brief, $600 \mathrm{ng}$ of labeled cRNA was combined with $5 \mu \mathrm{l} 10 \mathrm{X}$ blocking agent, brought to $24 \mu \mathrm{l}$ with $\mathrm{dd}_{2} \mathrm{O}$, and then combined with $1 \mu \mathrm{l}$ of $25 \mathrm{X}$ fragmentation buffer. The cRNA was then fragmented for exactly $30 \mathrm{~min}$ at $60^{\circ} \mathrm{C}$, cooled on ice for $1 \mathrm{~min}$, and then combined with $25 \mu 1$ of $2 \mathrm{X}$ GEx Hybridization Buffer HI-RPM by pipetting. The mix was centrifuged briefly and hybridized immediately to the microarrays. Arrays were rotated overnight for $17 \mathrm{~h}$ at $6^{\circ} \mathrm{C}$ at a high speed of rotation. 
Table I. Genes expressed in the responders in both the S-1 and UFT groups with cDNA microarray.

\begin{tabular}{lllccccc}
\hline Probe name & Gene symbol & $\begin{array}{c}\text { Genbank } \\
\text { accession no. }\end{array}$ & Regulation & P-value & $\begin{array}{c}\text { S-1 group } \\
\text { (n=30) } \\
\text { fold-change }\end{array}$ & $\begin{array}{c}\text { P-value } \\
\text { (n=29) } \\
\text { fold-change }\end{array}$ \\
\hline A_23_P134041 & TBX18 & NM_001080508 & Up & 0.013 & 2.28 & 0.018 & 2.72 \\
A_23_P7412 & BTNL8 & NM_001040462 & Down & 0.015 & 4.98 & 0.011 & 5.58 \\
A_24_P187056 & LOC375010 & AK090412 & Down & 0.007 & 2.73 & 0.029 & 2.19 \\
A_33_P3353737 & ADH1B & NM_000668 & Down & 0.009 & 2.53 & 0.049 & 2.37 \\
A_23_P105012 & HRASLS2 & NM_017878 & Down & 0.030 & 2.53 & 0.023 & 3.04 \\
A_33_P3282359 & LOC284232 & NR_027995 & Down & 0.008 & 2.40 & 0.037 & 2.34 \\
A_23_P420209 & GCNT3 & NM_004751 & Down & 0.041 & 2.27 & 0.011 & 4.18 \\
A_32_P13151 & LOC284232 & NR_027995 & Down & 0.028 & 2.20 & 0.031 & 2.24 \\
A_24_P73577 & ALDH1A2 & NM_170697 & Down & 0.050 & 2.13 & 0.010 & 2.32 \\
\hline
\end{tabular}

UFT, tegafur-uracil; S-1, tegafur/gimeracil/oteracil.

Arrays were disassembled in an ozone-filtered room using GE Wash Buffer I, washed for $1 \mathrm{~min}$ at room temperature and then washed for 1 min with pre-warmed $\left(37^{\circ} \mathrm{C}\right) \mathrm{GE}$ Wash Buffer II. The arrays were scanned immediately using an Agilent Microarray Scanner (Agilent).

miRNA microarray. Cyanine-3-labeled miRNA was prepared from $100 \mathrm{ng}$ total RNA using Agilent miRNA Complete Labeling and Hyb kit (Agilent) according to the manufacturer's instructions. Labeled miRNA was hybridized to Agilent Human miRNA Microarrays version 3, release $12.0(\mathrm{P} / \mathrm{N}$ G4471A, AMADID 021827) annotated against the Sanger miRbase 12.0 database of miRNAs, according to the manufacturer's instructions. The arrays were scanned immediately using an Agilent Microarray Scanner.

Data analysis. Data were analyzed using GeneSpring version 10 (Agilent). Among the 30 training samples in the $\mathrm{S}-1$ group, 18 were classified as responders and 12 as the non-responders. Among the 29 training samples in the UFT group, 15 were classified as responders and 14 as non-responders. Gene expression profiles were compared and fold-change values calculated to compare the responders and non-responders in both groups and identify predictive markers for patient responses to preoperative CRT. Genes that were differentially expressed were clustered.

Ingenuity pathway analysis. An ingenuity pathway analysis (IPA) (Ingenuity Systems, Mountain View, CA, USA) was used to identify biological and molecular networks that were related to the efficacy of the use of preoperative CRT. A detailed description is given in the online repository (http://www.ingenuity.com).

Statistical analysis. A total of 60 patients were required to have a power of 90 and a $5 \%$ single-sided significance level for the detection of a $25 \%$ increase in response rate in the combined experimental arm. The expression patterns in both the cDNA and miRNA microarrays were compared using unpaired t-tests. All differences were considered statistically significant at a p-value $<0.05$. All statistical analyses were performed using GeneSpring version 10.

\section{Results}

Response to CRT. Patient responses to CRT were evaluated with RECIST guidelines. One patient (3\%) in the S-1 group was determined to have had a complete response (CR); no patients in the UFT group showed a complete response. A partial response (PR) was noted in 17 patients $(57 \%)$ in the S-1 group and 15 patients $(52 \%)$ in the UFT group. Stable disease (SD) was determined in 12 patients $(40 \%)$ in the S-1 group and 14 patients (48\%) in the UFT group. No patient demonstrated progressive disease (PD). There was no statistically significant difference between the two groups in regard to response rate (CR and $\mathrm{PR} ; \mathrm{p}=0.52)$. For the purposes of the present study, patients were classified as responders (CR and $\mathrm{PR}$ ) or non-responders (SD and PD).

DNA microarray. Gene expression profiling was performed using customized and focused DNA microarrays. In the S-1 group, 184 genes were identified that were significantly $(\mathrm{p}<0.05)$ differentially expressed between the responders and the non-responders (data not shown). In the UFT group, 193 genes were identified that were significantly $(\mathrm{p}<0.05)$ differentially expressed between the responders and the nonresponders (data not shown). TBX18 upregulation was common among the responders in both groups, whereas reduced expression of BTNL8, LOC375010, ADH1B, HRASLS2, LOC284232, GCNT3 and ALDH1A2 was common to responders in both the S-1 and UFT groups (Table I). The results of a hierarchical cluster analysis are presented in Fig. 2. The responders and non-responders were not clustered in both groups.

Ingenuity pathway analysis. To identify predictive genes for preoperative CRT, an Ingenuity pathway analysis (IPA) was performed (Fig. 3). The IPA showed that the 'Infection Mechanism' network and the 'Endocrine System' network were related to responders in the S-1 group. The 'Infection 

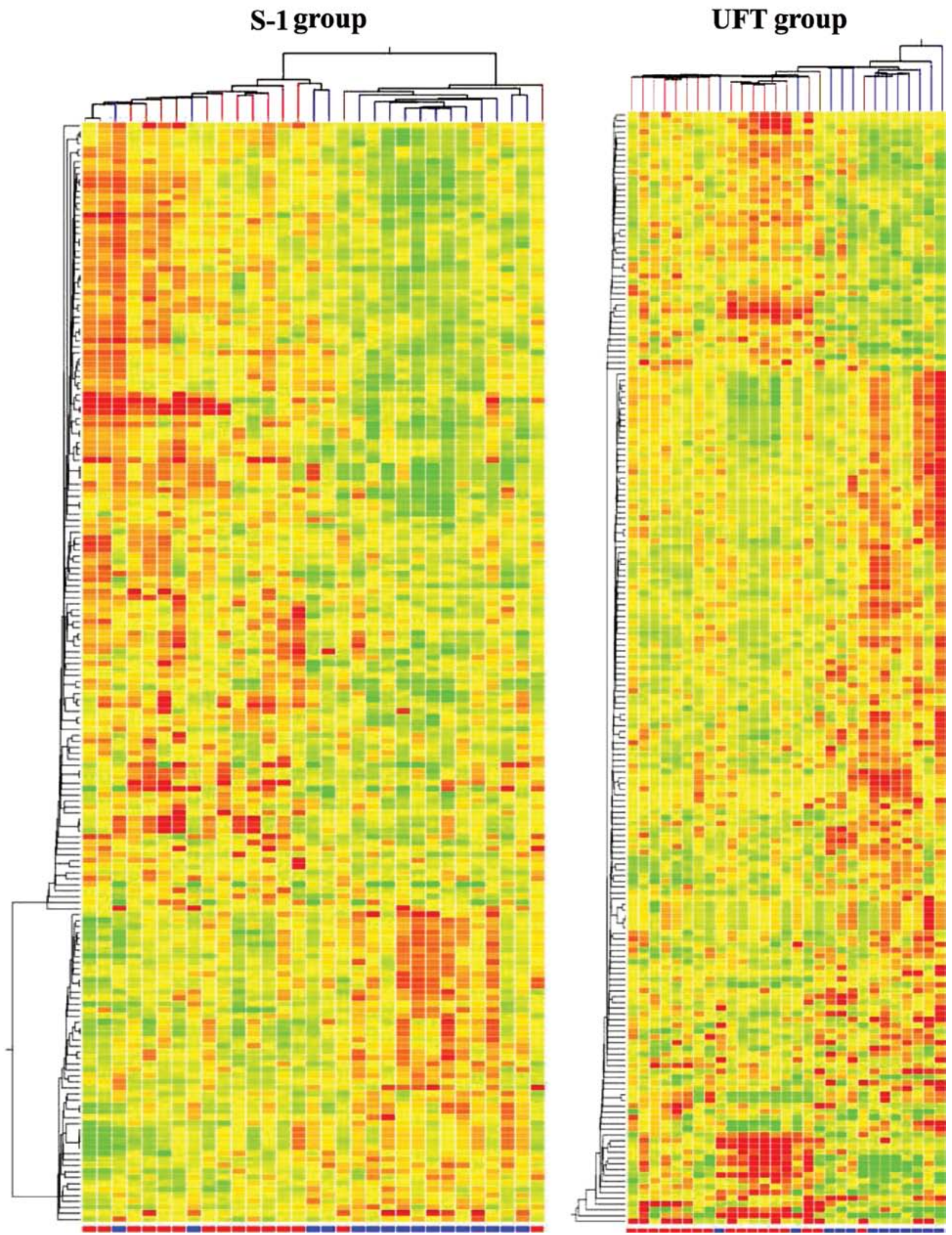

Figure 2. Hierarchical cluster analysis of 184 genes in the S-1 group and 194 genes in the UFT group. Top, overexpression is indicated in red and reduced expression in green. Bottom, non-responders are indicated in red; responders in blue. Patients were not clustered. UFT, tegafur-uracil; S-1, tegafur/gimeracil/oteracil.

Mechanism' network comprises chemokine genes and collagen genes such as CXC chemokine, TNFRSF6B, GREM1 and collagen, among others. These genes were significantly elevated in the S-1 responders, as determined using DNA microarray.
Comparatively, the 'Infection Mechanism' network and the 'Cancer Cell Signaling' network were related to responders in the UFT group, with elevated expression of the CXC chemokine and collagen from the 'Infection Mechanism' network. 
A

\section{S-1 group}

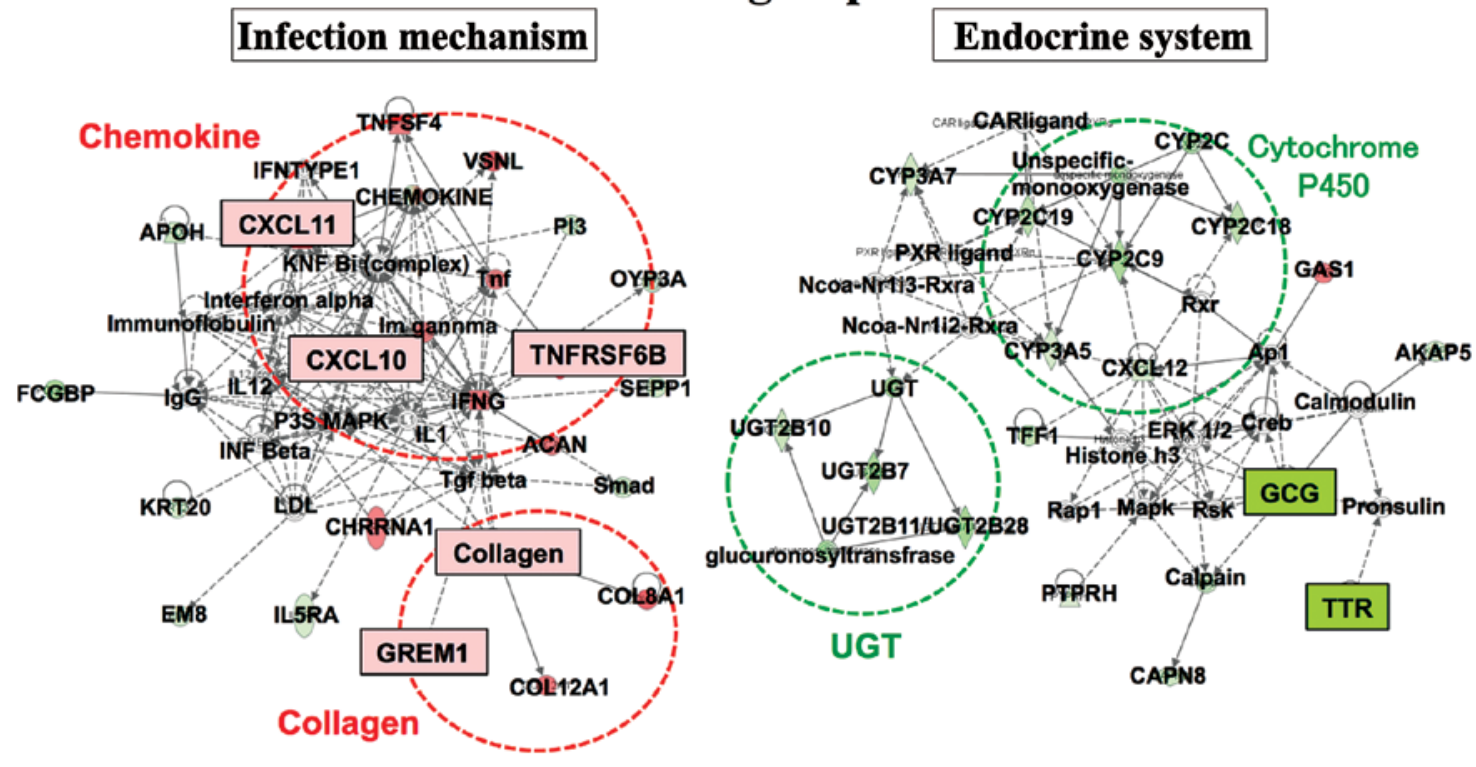

B

Infection mechanism

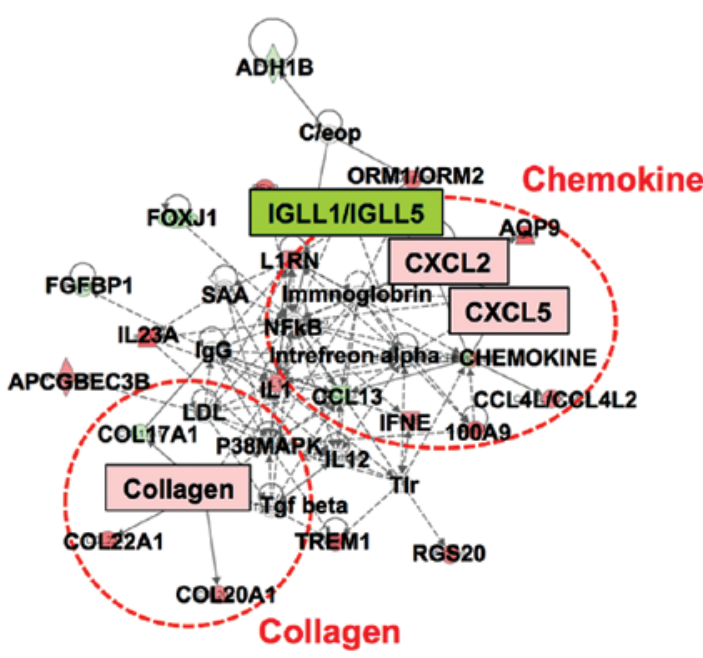

UFT group

\section{Cancer cell signaling}

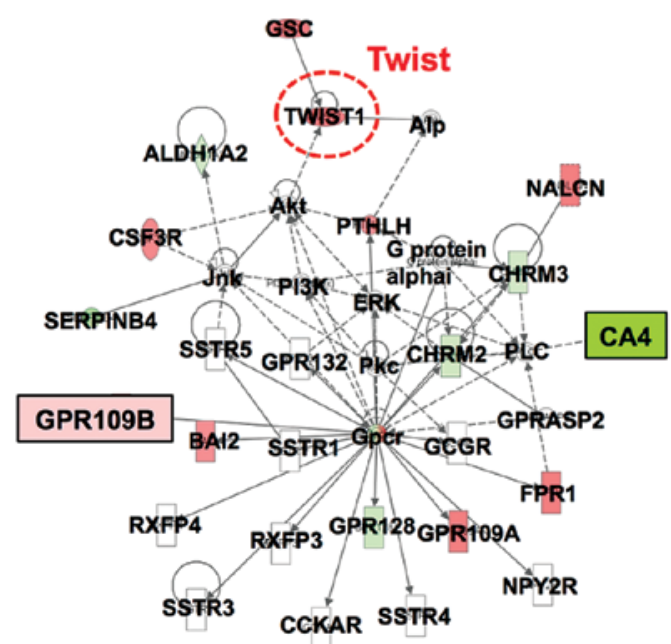

Figure 3. Ingenuity pathway analysis (IPA) for the S-1 group shows that 'Infection Mechanism' and 'Endocrine System' networks are related to the responders. The 'Infection Mechanism' network contains chemokine and collagen genes that were also elevated in the cDNA microarray analysis. IPA for the UFT group shows that 'Infection Mechanism' and 'Cancer Cell Signaling' networks are related to the responders. CXC chemokine and collagen, which were highly expressed in the DNA microarray, are included in the 'Infection Mechanism' network. UFT, tegafur-uracil; S-1, tegafur/gimeracil/oteracil.

miRNA microarray. The miRNA microarray showed significant $(\mathrm{p}<0.05)$ differential expression of six genes between the responders and non-responders in the S-1 group, with all six genes (miR-19-3p, miR-866-3p, miR-923, miR-494, miR-513a-5p and miR-513b) elevated in the responders (Table II). In contrast, 16 genes were significantly differentially expressed between the responders and nonresponders in the UFT group, with all 16 genes (miR-154, miR-379, miR-223, miR-1542-5p, miR-144, miR-363, miR-31, miR-1290, miR-382, miR-193a-5p, miR-451, miR-335, miR486-5p, miR-1246, miR-34b* and miR-144*) elevated in the responders. The results of a hierarchical cluster analysis are presented in Fig. 4. Again, the responders and non-responders were not clustered in both groups.

\section{Discussion}

Changes in gene expression patterns have been used to predict patient outcomes in multiple types of cancer. In the present study, 184 and 193 genes were differentially expressed between the responders and the non-responders in the patients treated with preoperative CRT using S-1 (S-1 group) and UFT (UFT group), respectively. TBX18 upregulation and BTNL8, LOC375010, ADH1B, HRASLS2, LOC284232, GCNT3 and ALDH1A2 downregulation were common to the responders from both treatment groups. Previous studies have also reported significant differential gene expression between responders and non-responders using preoperative CRT $(6,8)$, yet few common genes could be identified between different 

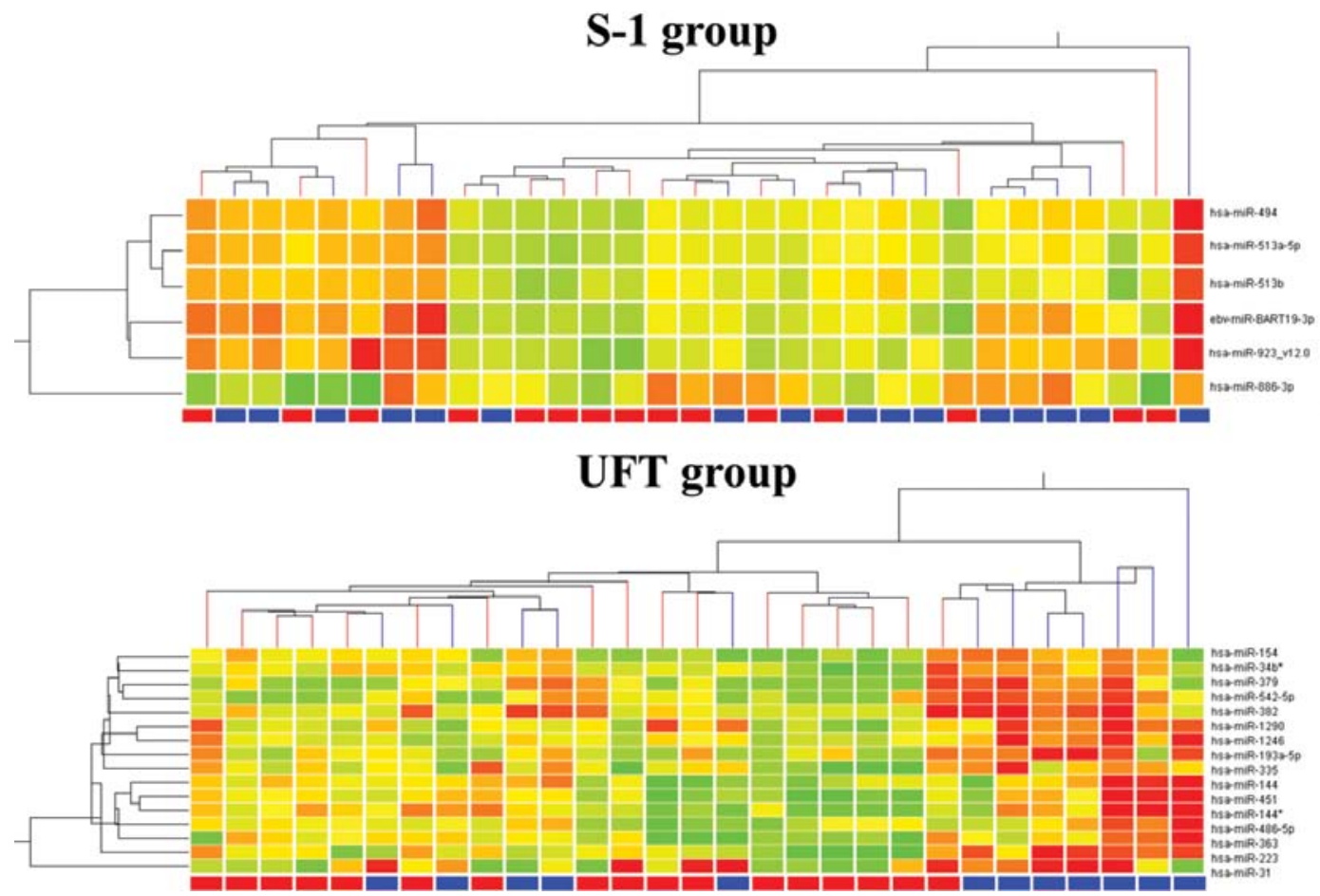

Figure 4. Hierarchical cluster analysis of six genes in the S-1 group and 16 genes in the UFT group. Top: Overexpression is indicated in red and reduced expression in green. Bottom, non-responders are indicated in red; responders in blue. Patients were not clustered. UFT, tegafur-uracil; S-1, tegafur/gimeracil/oteracil.

Table II. Genes expressed in the responders in the S-1 and UFT groups with miRNA microarray.

\begin{tabular}{lccc}
\hline Systematic name & P-value & Fold-change & Regulation \\
\hline $\begin{array}{l}\text { S-1 group (n=30) } \\
\text { hsa-miR-494 }\end{array}$ & 0.007 & 1.74 & $\mathrm{Up}$ \\
ebv-miR-BART19-3 & 0.007 & 2.20 & $\mathrm{Up}$ \\
hsa-miR-513a-5p & 0.010 & 1.59 & $\mathrm{Up}$ \\
hsa-miR-886-3p & 0.050 & 2.07 & $\mathrm{Up}$ \\
hsa-miR-923_v12.0 & 0.040 & 1.80 & $\mathrm{Up}$ \\
hsa-miR-513b & 0.027 & 1.51 & $\mathrm{Up}$ \\
UFT group (n=29) & & & \\
hsa-miR-154 & 0.021 & 2.01 & $\mathrm{Up}$ \\
hsa-miR-379 & 0.013 & 2.41 & $\mathrm{Up}$ \\
hsa-miR-223 & 0.017 & 2.84 & $\mathrm{Up}$ \\
hsa-miR-542-5p & 0.010 & 2.33 & $\mathrm{Up}$ \\
hsa-miR-144 & 0.018 & 2.69 & $\mathrm{Up}$ \\
hsa-miR-363 & 0.022 & 2.28 & $\mathrm{Up}$ \\
hsa-miR-31 & 0.025 & 3.37 & $\mathrm{Up}$ \\
hsa-miR-1290 & 0.012 & 2.24 & $\mathrm{Up}$ \\
hsa-miR-382 & 0.034 & 2.01 & $\mathrm{Up}$ \\
hsa-miR-193a-5p & 0.021 & 2.14 & $\mathrm{Up}$ \\
hsa-miR-451 & 0.006 & 3.30 & $\mathrm{Up}$ \\
hsa-miR-335 & 0.030 & 2.46 & $\mathrm{Up}$ \\
hsa-miR-486-5p & 0.008 & 2.18 & $\mathrm{Up}$ \\
hsa-miR-1246 & 0.002 & 2.31 & $\mathrm{Up}$ \\
hsa-miR-34b* & 0.028 & 2.02 & $\mathrm{Up}$ \\
hsa-miR-144* & 0.026 & 2.69 & $\mathrm{Up}$ \\
\hline
\end{tabular}

UFT, tegafur-uracil; S-1, tegafur/gimeracil/oteracil. treatment regimes. Furthermore, although some studies have reported the use of microarrays to predict the responses of patients with rectal cancer to CRT using preoperative biopsy tissue samples, this is the first study to date to use both cDNA and miRNA microarray analyses to predict patient responses. Therefore, from our results, we recommend the use of preoperative CRT in patients who show a differential expression in any of these eight common differentially expressed genes in biopsy specimens collected during colonoscopic examination for rectal cancer. Moreover, the use of S-1 or UFT is preferred if genes specific to that treatment identified in the present study are also identified from the patient biopsies.

An IPA was preformed to identify predictive genes for preoperative CRT. In the present study, IPA showed that CXC chemokine and collagen, which are part of the 'Infection Mechanism' network, were related to responders in the S-1 and UFT groups. A previous report indicated that CXCR4 and CXCL12 were predictive factors of distant recurrence and poor prognosis in patients with rectal cancer who were treated with preoperative CRT (14). This result supports our finding that the CXC chemokine may be a predictive factor of response to preoperative CRT in advanced rectal cancer.

Using microRNA analyses, we found 16 genes to be significantly elevated in the responders as compared with the non-responders in the UFT group. miR-223 was among these elevated genes. In the S-1 group, six genes were significantly higher in the responders than in the non-responders and, although not significant, miR-223 expression showed a tendency towards elevated expression $(\mathrm{p}=0.06)$. Previous studies have linked miR-223 with malignant prognosis in colorectal, gastric and breast cancers and in esophageal squamous cell carcinoma (15-18). Another study showed that overexpression of miR-223 downregulates the expression of 
ataxia telangiectasia mutated (ATM) gene and sensitizes U87 cells to radiation in vitro and in vivo (19). We also previously reported that miR-223 was higher in responders as compared with non-responders in patients with rectal cancer who were treated with preoperative CRT using S-1 (20). Collectively, these results support our hypothesis that miR-223 may be a predictive factor in ascertaining the response of patients with rectal cancer to preoperative CRT. In cases where miR-223 expression changes are noted in biopsy specimens, we also recommend the use of preoperative CRT. Moreover, if certain miRNAs are upregulated (miR-494, miR-BART19-3, miR-513a-5p, miR-886-3p, miR-923 and miR-513b), it is better to use S-1 as an agent for CRT. The reciprocal is true for the identification of certain miRNAs (miR-154, miR-379, miR542-5p, miR-144, miR-363, miR-31, miR-1290, miR-382, miR-193a-5p, miR-451, miR-335, miR-486-5p, miR-1246, miR-34b and miR-144) that were upregulated in the UFT group.

In conclusion, cDNA and miRNA expression patterns offer the opportunity to identify potential new biomarkers to predict the response of patients with rectal cancer to preoperative CRT and will likely be useful in establishing individualized therapies for these patients.

\section{Acknowledgements}

The present study was partly financed by the Research Support Foundation of the University of Tokushima and Taiho Pharmaceutical, Co., Ltd. (research funding provided to Professor Mitsuo Shimada).

\section{References}

1. Sauer R: Adjuvant and neoadjuvant radiotherapy and concurrent radiochemotherapy for rectal cancer. Pathol Oncol Res 8: 7-17, 2002.

2. Gérard JP, Conroy T, Bonnetain F, Bouché O, Chapet O, ClosonDejardin MT, Untereiner M, Leduc B, Francois E, Maurel J, et al: Preoperative radiotherapy with or without concurrent fluorouracil and leucovorin in T3-4 rectal cancers: Results of FFCD 9203. J Clin Oncol 24: 4620-4625, 2006.

3. Bosset JF, Collette L, Calais G, Mineur L, Maingon P, RadosevicJelic L, Daban A, Bardet E, Beny A, Ollier JC; EORTC Radiotherapy group Trial 22921: Chemotherapy with preoperative radiotherapy in rectal cancer. N Engl J Med 355: 1114-1123, 2006.

4. Morimoto S, Shimada M, Kurita N, Sato H, Iwata T, Nishioka M, Yoshikawa K, Miyatani T, Kashihara H, Takasu C, et al: Preoperative radiotherapy combined with $\mathrm{S}-1$ for advanced lower rectal cancer: Phase I trial. Hepatogastroenterology 59: 1428-1432, 2012.

5. Hotchi M, Okitsu H, Miura M, et al: A phase II trial of preoperative chemoradiotherapy with oral DPD-inhibitory fluoropyrimimidines in patients with advanced rectal cancer. J Cancer Ther 3: 989-995, 2012.

6. Ghadimi BM, Grade M, Difilippantonio MJ, Varma S, Simon R, Montagna C, Füzesi L, Langer C, Becker H, Liersch T, et al: Effectiveness of gene expression profiling for response prediction of rectal adenocarcinomas to preoperative chemoradiotherapy. $\mathrm{J}$ Clin Oncol 23: 1826-1838, 2005.
7. Kim IJ, Lim SB, Kang HC, Chang HJ, Ahn SA, Park HW, Jang SG, Park JH, Kim DY, Jung KH, et al: Microarray gene expression profiling for predicting complete response to preoperative chemoradiotherapy in patients with advanced rectal cancer. Dis Colon Rectum 50: 1342-1353, 2007.

8. Rimkus C, Friederichs J, Boulesteix AL, Theisen J, Mages J, Becker K, Nekarda H, Rosenberg R, Janssen KP and Siewert JR: Microarray-based prediction of tumor response to neoadjuvant radiochemotherapy of patients with locally advanced rectal cancer. Clin Gastroenterol Hepatol 6: 53-61, 2008.

9. Watanabe T, Kobunai T, Akiyoshi T, Matsuda K, Ishihara S and Nozawa K: Prediction of response to preoperative chemoradiotherapy in rectal cancer by using reverse transcriptase polymerase chain reaction analysis of four genes. Dis Colon Rectum 57: 23-31, 2014.

10. Pazdur R, Hoff PM, Medgyesy D, Royce M and Brito R: The oral fluorouracil prodrugs. Oncology 12 (Suppl 7): S48-S51, 1998

11. Giralt J, Tabernero J, Navalpotro B, Capdevila J, Espin E, Casado E, Mañes A, Landolfi S, Sanchez-Garcia JL, de Torres I, et al: Pre-operative chemoradiotherapy with UFT and Leucovorin in patients with advanced rectal cancer: A phase II study. Radiother Oncol 89: 263-269, 2008.

12. Van Cutsem E,HoffPM,Harper P,Bukowski RM,CunninghamD, Dufour P, Graeven U, Lokich J, Madajewicz S, Maroun JA, et al: Oral capecitabine vs intravenous 5-fluorouracil and leucovorin: Integrated efficacy data and novel analyses from two large, randomised, phase III trials. Br J Cancer 90: 1190-1197, 2004.

13. Lee EM, Hong YS, Kim KP, Lee JL, Kim SY, Park YS, Choi DH, Kim JH, Lim SB, Yu CS, et al: Phase II study of preoperative chemoradiation with S-1 plus oxaliplatin in patients with locally advanced rectal cancer. Cancer Sci 104: 111-115, 2013.

14. Saigusa S, Toiyama Y, Tanaka K, Yokoe T, Okugawa Y, Kawamoto A, Yasuda $\mathrm{H}$, Inoue $\mathrm{Y}$, Miki $\mathrm{C}$ and Kusunoki $\mathrm{M}$ : Stromal CXCR4 and CXCL12 expression is associated with distant recurrence and poor prognosis in rectal cancer after chemoradiotherapy. Ann Surg Oncol 17: 2051-2058, 2010.

15. Zhang J, Luo X, Li H, Yue X, Deng L, Cui Y and Lu Y: MicroRNA-223 functions as an oncogene in human colorectal cancer cells. Oncol Rep 32: 115-120, 2014.

16. Li X, Zhang Y, Zhang H, Liu X, Gong T, Li M, Sun L, Ji G, Shi Y, Han Z, et al: miRNA-223 promotes gastric cancer invasion and metastasis by targeting tumor suppressor EPB41L3. Mol Cancer Res 9: 824-833, 2011.

17. Kurashige J, Watanabe M, Iwatsuki M, Kinoshita K, Saito S, Hiyoshi Y, Kamohara H, Baba Y, Mimori K and Baba H: Overexpression of microRNA-223 regulates the ubiquitin ligase FBXW7 in oesophageal squamous cell carcinoma. Br J Cancer 106: 182-188, 2012.

18. Pinatel EM, Orso F, Penna E, Cimino D, Elia AR, Circosta P, Dentelli P, Brizzi MF, Provero P and Taverna D: miR-223 is a coordinator of breast cancer progression as revealed by bioinformatics predictions. PLoS One 9: e84859, 2014.

19. Liang L, Zhu J, Zaorsky NG, Deng Y, Wu X, Liu Y, Liu F, Cai G, $\mathrm{Gu}$ W, Shen L, et al: MicroRNA-223 enhances radiation sensitivity of U87MG cells in vitro and in vivo by targeting ataxia telangiectasia mutated. Int J Radiat Oncol Biol Phys 88: 955-960, 2014.

20. Hotchi M, Shimada M, Kurita N, Iwata T, Sato H, Morimoto S, Yoshikawa K, Higashijima J and Miyatani T: microRNA expression is able to predict response to chemoradiotherapy in rectal cancer. Mol Clin Oncol 1: 137-142, 2013. 